\title{
An alternative subtraction scheme for NLO QCD calculations using Nagy-Soper dipoles
}

\author{
Markus Bach, Tania Robens* \\ IKTP, TU Dresden \\ E-mail: Markus.Bach1@mailbox.tu-dresden.de, \\ Tania.Robensetu-dresden.de \\ Cheng Han Chung \\ Supercomputing Research Center, National Cheng Kung University \\ E-mail: chenghan@mail.ncku.edu.tw
}

In this work, we briefly report on the current status of an alternative subtraction scheme which is based on the splitting kernels of an improved parton shower prescription. Our focus is here on more recent developments as well as generic arguments on the scaling behaviour.

11th International Symposium on Radiative Corrections (Applications of Quantum Field Theory to Phenomenology)

22-27 September 2013

Lumley Castle Hotel, Durham, UK

\footnotetext{
* Speaker.
} 


\section{Introduction: subtraction schemes in the NLO era}

It is indisputable that higher order corrections are needed to correctly predict fully differential distributions for scattering processes at high precision. The recent discovery of a Higgs boson [1], 2], which led to the award of this year's Nobel prize [3], is a more than intriguing example that precision physics is indispensable to a correct theoretical interpretation of experimental data accumulated at present and future colliders. An ideal framework for a detailed comparison between theoretical predictions and experimental findings are Monte Carlo event generators. In addition, especially in recent years many tools have been developed which allow for the (semi-)automated calculation of higher order corrections. These tools provide the virtual contributions, whereas the integration over phase space as well as the calculation of the real emission part is usually performed by a standard Monte Carlo generator, as e.g. Sherpa [ 4 ] or Herwig++ [5]. The next-to-leading order (NLO) matching of such higher order calculations to parton showers is equally well understood.

The implementation of NLO calculations into numerical tools exhibits a caveat stemming from the infrared divergence of real and virtual NLO contributions, which originate from different phase spaces: although in the sum of all contributions, the infinite parts exactly cancel, the behaviour of the divergence needs to be parametrized, e.g. by infinitesimal regulators. The implementation of such regulators into numerical codes can result in large unphysical numerical uncertainties. A way to circumvent this problem is the introduction of subtraction schemes, which efficiently reshuffle the divergent terms such that a numerically stable evaluation becomes possible for contributions stemming from both Born-type and real-emission kinematics. We here discuss a specific scheme and its properties, which has first been proposed in [6], using splitting kernels as well as mapping prescriptions which were already suggested in the framework of an improved parton shower [7, 8 , 91. It was further developed for processes with an arbitrary number of final states in [10], and a recent review was presented in [11]. Furthermore, the scheme has recently been implemented in an automated way within the HelacNLO framework [12]. We here largely follow the notation of [6, 10, 11] and only briefly review the setup of the scheme, rather focussing on newer developments which have not been presented previously. We equally reemphasize a theoretical argument [10] which in principle allows for an even further reduction of the scaling.

\section{Subtraction Schemes}

Higher order subtraction schemes make use of factorization of the real-emission matrix element in the soft or collinear limits, leading to the decomposition $\left|\mathscr{M}_{m+1}(\hat{p})\right|^{2} \longrightarrow \mathscr{D}_{\ell} \otimes\left|\mathscr{M}_{m}(p)\right|^{2}$ [13, 14, 15]. Here, $\mathscr{D}_{\ell}$ are the dipoles which contain the respective singularity structure. The symbol $\otimes$ denotes a correct convolution in colour, spin, and flavour space, and $\hat{p} / p$ are momenta in $(m+1) / m$-parton phase space. The subtracted contributions are then given by

$$
\sigma^{\mathrm{NLO}}=\underbrace{\int_{m+1}\left[d \sigma^{R}-d \sigma^{A}\right]}_{\text {finite }}+\underbrace{\int_{m+1} d \sigma^{A}+\int_{m} d \sigma^{V}}_{\text {finite }}
$$


where

$$
\begin{aligned}
\int_{m}\left[d \sigma^{B}+d \sigma^{V}+\int_{1} d \sigma^{A}\right] & =\int d P S_{m}\left[\left|\mathscr{M}_{m}\right|^{2}+\left|\mathscr{M}_{m}\right|_{\text {one-loop }}^{2}+\sum_{\ell} \mathscr{V}_{\ell} \otimes\left|\mathscr{M}_{m}\right|^{2}\right], \\
\int_{m+1}\left[d \sigma^{R}-d \sigma^{A}\right] & =\int d P S_{m+1}\left[\left|\mathscr{M}_{m+1}\right|^{2}-\sum_{\ell} D_{\ell} \otimes\left|\mathscr{M}_{m}\right|^{2}\right],
\end{aligned}
$$

and where $\int d P S$ denotes the integration over the respective phase space, including all symmetry and flux factors. The symbols $d \sigma^{B}, d \sigma^{V}, d \sigma^{R}$ stand for the Born, virtual and real-emission contributions of the calculation, while real-emission subtraction terms are summarized as $d \sigma^{A}$. Since $\left|\mathscr{M}_{m+1}\right|^{2}$ and $\left|\mathscr{M}_{m}\right|^{2}$ live in different phase spaces, their momenta need to be mapped via a mapping function. Furthermore, the subtraction term $\mathscr{D}_{\ell}$ and its one-parton integrated counterpart $\mathscr{V}_{\ell}$ are related by $\mathscr{V}_{\ell}=\int d \xi_{p} \mathscr{D}_{\ell}$, where $d \xi_{p}$ is an unresolved one-parton integration measure. The following ingredients therefore define a subtraction scheme: (a) a suitable mapping from $(m+1)$ to $m$ parton phase space which guarantees energy-momentum conservation as well as on-shellness, and (b) an efficient parametrization of the one-parton integration measure $d \xi_{p}$. While the number of reevaluations of the underlying Born matrix element for the real emission subtractions in Eqn. (2.2) is determined by (a), the complexity of the integrated counterterms depends on (b). Currently, two major schemes for NLO subtraction are on the market, namely the Catani-Seymour (CS) dipole scheme [16, 17], and the Frixione-Kunszt-Signer (FKS) subtraction [18]. In the scheme discussed here, we use the splitting kernels of an improved parton shower as a basis for the real emission subtraction terms ${ }^{1}$, and we apply a momentum mapping which leads to an overall scaling behaviour $\sim N^{2}$ for a process with $N$ partons in the final state. The number of matrix element reevaluations is thereby reduced by a factor proportional to the number of final state particles of the process with respect to the CS scheme.

\section{Nagy-Soper subtraction: Setup and relation to improved parton shower}

We denote four-momenta in the Born-type kinematics by unhatted quantities $p_{i}$, while the real emission phase space momenta are denoted by hatted quantities $\hat{p}_{i}$; initial state momenta are labelled $p_{a}$ and $p_{b}$, where $Q=p_{a}+p_{b}$ and with $Q^{2}$ being the squared centre-of-mass energy, with equivalent relations in the real emission phase space; generally, $\hat{p}_{\ell}$ labels the emitter, $\hat{p}_{j}$ the emitted parton and $\hat{p}_{k}$ the spectator.

\subsection{Scheme setup}

The scheme discussed here uses the splitting kernels of an improved parton shower [7, 8, 9] as a basis for the subtraction terms. We can therefore write [7]

$$
\left|\mathscr{M}_{\ell}\left(\{\hat{p}, \hat{f}\}_{m+1}\right)\right\rangle=t_{\ell}^{\dagger}\left(f_{\ell} \rightarrow \hat{f}_{\ell}+\hat{f}_{j}\right) V_{\ell}^{\dagger}\left(\{\hat{p}, \hat{f}\}_{m+1}\right)\left|\mathscr{M}\left(\{p, f\}_{m}\right)\right\rangle .
$$

Here, $\left|\mathscr{M}_{\ell}\left(\{\hat{p}, \hat{f}\}_{m+1}\right)\right\rangle$ and $\left|\mathscr{M}\left(\{p, f\}_{m}\right)\right\rangle$ denote the matrix elements in real emission $(m+1)$ and Born-type $(m)$ phase space and $V_{\ell}, t_{\ell}$ the factorization operators in colour and spin space. For

\footnotetext{
${ }^{1}$ This equally promises to facilitate the matching to the improved parton shower, cf. discussion in [1] and references therein.
} 
fermionic emitters, the splitting functions are diagonal in helicity space; therefore, the real emission subtraction terms are directly given by the spin averaged functions $\bar{W}_{\ell \ell}=\frac{1}{2} v_{\ell}^{2}$, with $v_{\ell}^{2}$ being defined by Eqn. (43) in [6]. In case of gluonic emitters, information on the gluon polarization needs to be retained, and soft/ collinear divergences from interference terms arise. In our scheme, these are treated using dipole partitioning functions $A_{\ell k}$ [9], which redistribute the singularities to contributions $W_{\ell k}^{(\ell)}, W_{\ell k}^{(k)}$, where $p_{\ell}, p_{k}$ take over the kinematic role of the mother parton in the mapping, respectively. The subtraction term is then split into a purely collinear and a soft/ collinear part $\left\langle v^{\prime}\left|W_{\ell \ell}-W_{\ell k}\right| v\right\rangle=\left\langle v^{\prime}\left|\left(W_{\ell \ell}-W_{\ell \ell}^{\text {eik }}\right)+\left(W_{\ell \ell}^{\text {eik }}-W_{\ell k}\right)\right| v\right\rangle$, where $|v\rangle,\left|v^{\prime}\right\rangle$ denote the gluon polarization of the mother parton connected to the Born-type matrix element in Eqn. (3.1). Our specific choice of the dipole partitioning functions leads to

$$
\Delta W_{\ell k}=\bar{W}_{\ell \ell}^{\mathrm{eik}}-\bar{W}_{\ell k}=4 \pi \alpha_{s} \frac{2\left(\hat{p}_{\ell} \cdot \hat{p}_{k}\right)\left(\hat{p}_{\ell} \cdot \hat{Q}\right)}{\left(\hat{p}_{\ell} \cdot \hat{p}_{j}\right)\left[\left(\hat{p}_{j} \cdot \hat{p}_{k}\right)\left(\hat{p}_{\ell} \cdot \hat{Q}\right)+\left(\hat{p}_{\ell} \cdot \hat{p}_{j}\right)\left(\hat{p}_{k} \cdot \hat{Q}\right)\right]}
$$

where $\bar{W}_{\ell \ell}^{\text {eik }}$ is the spin-averaged eikonal factor. All quantities are defined as in [6, 10, 11].

\subsection{Final state momentum mapping and scaling behaviour}

The improved scaling behaviour of our scheme mainly results from the specific mapping between the real emission and Born-type kinematic phase spaces for final state emitters. For final state mappings, we use the whole remainder of the event as a spectator in terms of momentum redistributions; therefore, we have

$$
p_{\ell}=\frac{1}{\lambda_{\ell}}\left(\hat{p}_{\ell}+\hat{p}_{j}\right)-\frac{1-\lambda_{\ell}+y_{\ell}}{2 \lambda_{\ell} a_{\ell}} Q, p_{n}^{\mu}=\Lambda(K, \hat{K})^{\mu}{ }_{v} \hat{p}_{n}^{v}, \quad n \notin\{\ell, j=m+1\},
$$

with $\Lambda(K, \hat{K})^{\mu}{ }_{v}=g^{\mu}{ }_{v}-\frac{2(K+\hat{K})^{\mu}(K+\hat{K})_{v}}{(K+\hat{K})^{2}}+\frac{2 K^{\mu} \hat{K}_{v}}{\hat{K}^{2}}$, where $y_{\ell}=\frac{P_{\ell}^{2}}{2 P_{\ell} \cdot Q-P_{\ell}^{2}}$. We furthermore introduced $\lambda_{\ell}\left(y_{\ell}, a_{\ell}\right)=\sqrt{\left(1+y_{\ell}\right)^{2}-4 a_{\ell} y_{\ell}}, K=Q-p_{\ell}, \hat{K}=Q-P_{\ell}, a_{\ell}\left(P_{\ell}, Q\right)=\frac{Q^{2}}{2 P_{\ell} \cdot Q-P_{\ell}^{2}}$, with $P_{\ell}=\hat{p}_{\ell}+$ $\hat{p}_{j}$. Note that it is the global mapping for all remaining particles in Eqn. (3.3) that is responsible for the reduced number of Born-type matrix reevaluations. For the real emission subtraction terms, we then obtain the total contribution

$$
d \sigma_{a b}^{A}\left(\hat{p}_{a}, \hat{p}_{b}\right)=d \sigma_{a b}^{A, a}\left(\hat{p}_{a}, \hat{p}_{b}\right)+d \sigma_{a b}^{A, b}\left(\hat{p}_{a}, \hat{p}_{b}\right)+\sum_{\ell \neq a, b} d \sigma_{a b}^{A, \ell}\left(\hat{p}_{a}, \hat{p}_{b}\right),
$$

with the sum over all possible final state emitters. For a specific emitter $\hat{p}_{\ell}$, it is explicitly given by

$$
\begin{aligned}
d \sigma_{a b}^{A, \ell}\left(\hat{p}_{a}, \hat{p}_{b}\right)= & \frac{N_{m+1}}{\Phi_{m+1}} \sum_{j}\left\{\left[\mathscr{D}_{g q q}\left(\hat{p}_{j}\right) \delta_{g ; q, q_{j}}+\mathscr{D}_{g g g}\left(\hat{p}_{j}\right) \delta_{g ; g, g_{j}}\right]\left|\mathscr{M}_{\mathrm{Born}, g}\right|^{2}\left(p_{a}, p_{b} ; p_{n}\right)\right. \\
& \left.+\left[\mathscr{D}_{q q g}\left(\hat{p}_{j}\right) \delta_{q ; g, q_{j}}+\mathscr{D}_{q q g}\left(\hat{p}_{j}\right) \delta_{q ; q, g_{j}}\right]\left|\mathscr{M}_{\mathrm{Born}, q}\right|^{2}\left(p_{a}, p_{b} ; p_{n}\right)\right\}
\end{aligned}
$$

where $\Phi_{m+1}$ denotes the flux factor ${ }^{2}$. The subtraction terms can be split into collinear and interference parts:

$$
\mathscr{D}_{f_{\ell} \hat{f}_{\ell} \hat{f}_{j}}\left(\hat{p}_{\ell}, \hat{p}_{j}\right)=\mathscr{D}_{f_{\ell} \hat{f}_{\ell} \hat{f}_{j}}^{\text {coll }}\left(\hat{p}_{\ell}, \hat{p}_{j}\right)+\delta_{\hat{f}_{j}, g} \sum_{k \neq(\ell, j)} \mathscr{D}^{\text {if }}\left(\hat{p}_{\ell}, \hat{p}_{j}, \hat{p}_{k}\right)
$$

\footnotetext{
${ }^{2}$ The $\delta_{f_{\ell} ; \hat{f}_{\ell} \hat{f}_{j}}$ functions ensure the existence of the respective splittings $f_{\ell} \rightarrow \hat{f}_{\ell} \hat{f}_{j}$ in flavour space.
} 
where $\mathscr{D}^{\text {if }}\left(\hat{p}_{\ell}, \hat{p}_{j}, \hat{p}_{k}\right)$ denotes an interference contribution with $\hat{p}_{k}$ acting as a spectator. Each of the contributions in Eqn. (3.6) requires exactly one global mapping, i.e. the number of mappings and thereby matrix reevaluations in the real emission subtraction terms behaves like $\sim \#(\ell j)$. This leads to an overall scaling behaviour $\sim N^{2} / 2$, where $N$ is the number of final state particles, which can in principle be improved even further. In [19], the authors show that within the MadFKS environment, a constant scaling behaviour can be achieved; i.e., for certain types of processes, the number of reevaluations of underlying Born-type matrix elements in the real-emission subtraction terms remains constant. This relies on the fact that any $m+1$ phase space can be decomposed into disjoint partitions that are specified by their behaviour for one of the partons $\hat{p}_{i}$ becoming soft or collinear to at most one other parton $\hat{p}_{j}$, where the sum of all FKS partitions reproduces the whole phase space ${ }^{3}$, such that $\sum_{(i, j) \in \mathscr{P}_{\mathrm{FKS}}} \mathscr{S}_{i j}=1$. Here, $\mathscr{P}_{\mathrm{FKS}}$ is the set of FKS pairs labelled by the parton indices $(i, j)$, and $\mathscr{S}_{i j}$ are the $S$-functions [19] which project out the respective FKS partition $(i, j)$. Furthermore, several partitions render exactly the same contribution to the final observable, and therefore the evaluation of only one of these is sufficient:

$$
d \sigma^{(n+1)}(r)=\sum_{(i, j) \in \overline{\mathscr{P}}_{\mathrm{FKS}}} \xi_{i j}^{(n+1)}(r) d \sigma_{i j}^{(n+1)}(r)
$$

where $\xi_{i j}^{(n+1)}(r)$ is the process-dependent symmetry factor that relates the total cross section to the

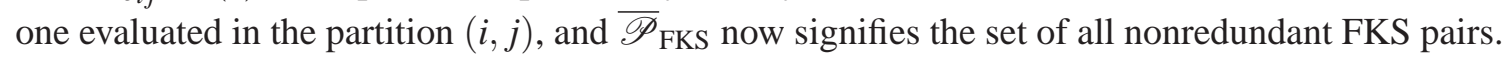
In the scheme discussed here, the subtraction term that reflects the divergences of $\mathscr{S}_{i j}$ is given by Eqn. (3.6), such that all contributions from the soft/ collinear divergence of $\hat{p}_{i}, \hat{p}_{k}$ are transferred to the interference term $\mathscr{D}^{\text {if }}\left(\hat{p}_{k}, \hat{p}_{i}, \hat{p}_{j}\right)$, corresponding to the singularity structure of a different partition, namely $\mathscr{S}_{i k}$. All terms in Eqn. (3.6) come with the same mapping, and, as in the FKS prescription in [19], only the set of nonredundant contributions needs to be evaluated, all others being related by symmetry. Increasing the number of final state gluons then leads to a change in the constant $\xi_{i j}^{(n+1)}(r)$ but does not call for the evaluation of a larger number of nonredundant contributions, as the number of elements in $\overline{\mathscr{P}}_{\text {FKS }}$ remains constant. Therefore, following this prescription, our scheme equally exhibits a constant scaling behaviour, when the number of gluons in the real emission final state is increased.

\subsection{Subtraction terms and integrated counterterms}

We devote this subsection to a more detailed discussion of one of the leftover finite parts in the integrated counterterms that is currently evaluated numerically. The existence of finite remainders in these terms is a direct consequence of the modified mapping which leads to the improved scaling behaviour discussed above. Although this constitutes a slight modification with respect to standard schemes such as CS and FKS, it poses no impediment for the implementation of our scheme.

We here present an example of an approximation for one of these integrals. For this, we focus on an integral which appears in the final state $q q g$ splitting, namely

$$
I_{3}\left(a_{\ell}\right)=-\int_{0}^{y_{\max }} d y\left[\frac{(\lambda-1+y)^{2}}{4 y}+1\right] \frac{(1+y) \ln x_{0}}{\lambda},
$$

\footnotetext{
${ }^{3}$ Note that the notation between [19] and this work differs in the fact that in [19], $\hat{p}_{i}$ labels the emitted parton that becomes soft or collinear, while in our case this parton is denoted by $\hat{p}_{j}$. For sake of consistency, we stick to the notation proposed in [19] in the above discussion.
} 

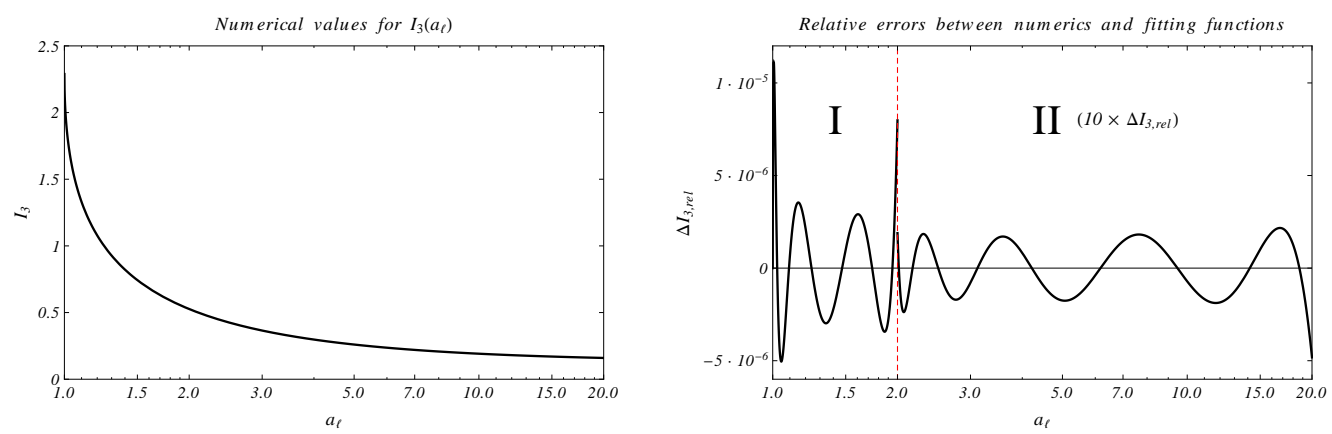

Figure 1: Left: Numerical values for $I_{3}\left(a_{\ell}\right)$ (Eqn. (3.8)), in the range $a_{\ell} \in[1,20]$. Right: Relative error between numerical value for $I_{3}\left(a_{\ell}\right)$ and fitting functions for $a_{\ell} \in[1,2]$ (section I) and $a_{\ell} \in[2,20]$ (section II), given by Eqns. (3.9) and (3.10) respectively. We find the relative errors are $\mathscr{O}\left(10^{-5}\right)$, thereby well below typical phase space integration errors. Note that in the above plot the relative error in section II has been multiplied by 10 for better visibility.

where $\lambda \equiv \lambda_{\ell}\left(y, a_{\ell}\right), x_{0}\left(y, a_{\ell}\right)=\frac{1-\lambda+y}{1+\lambda+y}, y_{\max }\left(a_{\ell}\right)=\left(\sqrt{a_{\ell}}-\sqrt{a_{\ell}-1}\right)^{2}$, and all other parameters are defined in Section 3.2. For $a_{\ell}=1$, the integral is given by $I_{3}(1)=\frac{\pi^{2}}{3}-1$; for all other cases, we use an approximation. We easily find the approximating functions

$$
\begin{aligned}
& I_{3}^{\text {approx }, a_{\ell} \leq 2}\left(a_{\ell}\right)=\frac{a_{\ell}}{4}+\left(1+a_{\ell}^{2}\right)\left[\frac{\pi^{2}}{6}-\operatorname{Li}_{2}\left(1-y_{\max }\right)\right]+2\left(a_{\ell}-1\right)^{2} \ln \left(1-y_{\max }\right) \\
& +\frac{1}{16}\left[13 y_{\max }\left(1-a_{\ell}\right)^{2}-5 a_{\ell}^{2} y_{\max }+\ln y_{\max }\left[5+4\left(a_{\ell}+2 y_{\max }-3 a_{\ell} y_{\max }\right)\right]-15\right] \\
& \quad-\frac{\left(a_{\ell}-1\right)\left(a_{\ell}^{2}-2.75128 a_{\ell}+1.74026\right)}{0.248486 a_{\ell}^{3}+7.10958 a_{\ell}^{2}-3.11175 a_{\ell}-3.68862} \\
& I_{3}^{\text {approx }, 2 \leq a_{\ell} \leq 20}\left(a_{\ell}\right)=0.12842+\frac{0.560353}{\left(a_{\ell}-0.578142\right)^{0.974664}} \\
& \quad+\frac{a_{\ell}^{4}-28.1242 a_{\ell}^{3}+240.479 a_{\ell}^{2}-758.744 a_{\ell}+778.706}{1457.74 a_{\ell}^{4}+16001.5 a_{\ell}^{3}-48392.7 a_{\ell}^{2}+64199 a_{\ell}-39009.3}
\end{aligned}
$$

For $a_{\ell} \geq 20$, a similar approximation applies.

Figure 1 shows the behaviour of the integral for the range $a_{\ell} \in[1,20]$, as well as the relative errors between the approximation as given in Eqns. (3.9), (3.10) and the numerically evaluated integral; we found that the errors are $\mathscr{O}\left(10^{-5}\right)$ for $a_{\ell} \in[1,2]$, and an order of magnitude smaller for $a_{\ell} \geq 2$. We want to emphasize that these relative errors are small compared to the errors typically obtained from the Monte Carlo integration over phase space.

\section{Results}

As an example, we here present results for the process $e^{+} e^{-} \rightarrow 3$ jets [10]. The leading order contribution is given by $e^{+} e^{-} \rightarrow q \bar{q} g$, and we include virtual corrections, as well as real emission processes $e^{+} e^{-} \rightarrow q \bar{q} q \bar{q}, e^{+} e^{-} \rightarrow q \bar{q} g g$ [20]. The above real emission contributions 

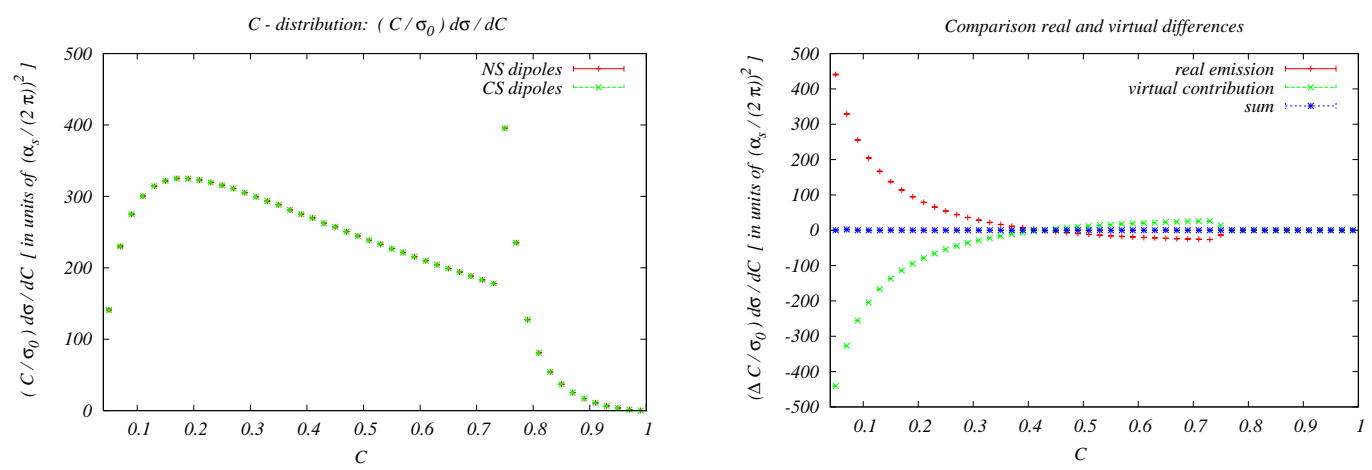

Figure 2: Left: Total result for differential distribution $\frac{C}{\sigma_{0}} \frac{d \sigma^{\mathrm{NLO}}}{d C}$ using both our scheme (labelled NS, red) and CS (green) dipoles. The standard literature result obtained using the CS scheme is completely reproduced with the NS dipoles. Right: Differences $\Delta_{\text {CS-NS }}$ for real emission (red, upper) and virtual (green, lower) contributions, showing that especially for low $C$ values the contributions in the two schemes significantly differ. Adding up $\Delta^{\text {real }}+\Delta^{\text {virt }}$ gives 0 as expected.

call for $(8+10)$ matrix element reevaluations per phase space point in the CS and $(4+5)$ reevaluations in our scheme, respectively. We display our results in terms of the $\mathrm{C}$ distribution [20] $C^{(n)}=3\left\{1-\sum_{i, j=1, i<j}^{n} \frac{s_{i j}^{2}}{\left(2 p_{i} \cdot Q\right)\left(2 p_{j} \cdot Q\right)}\right\},\left(s_{i j}=2 p_{i} \cdot p_{j}\right)$, which fulfills all requirements of a jet observable and is infrared finite on the integration level [21].

Figure 2 shows that we reproduce the literature result, numerically obtained from [22], and equally found agreement between implementations of both schemes. We want to emphasize that this is indeed a non-trivial statement, since the differences between the two schemes for both subtracted real emission as well as virtual contributions are sizeable; therefore, agreement between the two schemes on the per mil level [10] constitutes a non-trivial validation of our scheme.

\section{Summary}

We here reported on some recent progress in the development of an alternative NLO subtraction scheme for QCD calculations, which uses the splitting functions of an improved parton shower as subtraction kernels. We have briefly discussed the setup, and especially the features leading to an improved scaling behaviour of our scheme with respect to one of the standard subtraction schemes. We focussed on possible further improvements of this scaling behaviour, along the lines of a proposal which has first been investigated within the MadFKS framework. Results for the process $e^{+} e^{-} \rightarrow 3$ jets as well as an example of an analytic approximation for one of the functions which is currently evaluated numerically have been presented. Summarizing, we regard the scheme discussed here as a viable alternative to both CS and FKS subtraction. Our scheme exhibits a smaller number of subtraction terms with respect to CS, and does not call for a reparametrization of the phase space for each emitter/ emitted parton pair, as needed in the FKS scheme. We believe that this direction is worthwhile to investigate, and plan to implement this into a general purpose Monte Carlo event generator in the near future. 


\section{References}

[1] Georges Aad et al. Observation of a new particle in the search for the Standard Model Higgs boson with the ATLAS detector at the LHC. Phys.Lett., B716:1-29, 2012.

[2] Serguei Chatrchyan et al. Observation of a new boson at a mass of $125 \mathrm{GeV}$ with the CMS experiment at the LHC. Phys.Lett., B716:30-61, 2012.

[3] http://www.nobelprize.org/nobel_prizes/physics/laureates/2013/.

[4] T. Gleisberg, S. Hoeche, F. Krauss, M. Schonherr, S. Schumann, et al. Event generation with SHERPA 1.1. JHEP, 0902:007, 2009.

[5] M. Bahr, S. Gieseke, M.A. Gigg, D. Grellscheid, K. Hamilton, et al. Herwig++ Physics and Manual. Eur.Phys.J., C58:639-707, 2008.

[6] C.H. Chung, M. Kramer, and T. Robens. An alternative subtraction scheme for next-to-leading order QCD calculations. JHEP, 1106:144, 2011.

[7] Zoltan Nagy and Davison E. Soper. Parton showers with quantum interference. JHEP, 09:114, 2007.

[8] Zoltan Nagy and Davison E. Soper. Parton showers with quantum interference: leading color, spin averaged. JHEP, 03:030, 2008.

[9] Zoltan Nagy and Davison E. Soper. Parton showers with quantum interference: leading color, with spin. JHEP, 07:025, 2008.

[10] Cheng-Han Chung and Tania Robens. Nagy-Soper subtraction scheme for multiparton final states. Phys.Rev., D87:074032, 2013.

[11] Tania Robens. Nagy-Soper Subtraction: A Review. Mod.Phys.Lett., A28:1330020, 2013.

[12] G. Bevilacqua, M. Czakon, M. Kubocz, and M. Worek. Complete Nagy-Soper subtraction for next-to-leading order calculations in QCD. JHEP, 1310:204, 2013.

[13] Guido Altarelli and G. Parisi. Asymptotic Freedom in Parton Language. Nucl. Phys., B126:298, 1977.

[14] A. Bassetto, M. Ciafaloni, and G. Marchesini. Jet Structure and Infrared Sensitive Quantities in Perturbative QCD. Phys. Rept., 100:201-272, 1983.

[15] Yuri L. Dokshitzer, Valery A. Khoze, Alfred H. Mueller, and S. I. Troian. Basics of perturbative QCD. Gif-sur-Yvette, France: Ed. Frontieres (1991) 274 p. (Basics of).

[16] S. Catani and M. H. Seymour. A general algorithm for calculating jet cross sections in nlo qcd. Nucl. Phys., B485:291-419, 1997.

[17] Stefano Catani, Stefan Dittmaier, Michael H. Seymour, and Zoltan Trocsanyi. The dipole formalism for next-to-leading order QCD calculations with massive partons. Nucl. Phys., B627:189-265, 2002.

[18] S. Frixione, Z. Kunszt, and A. Signer. Three jet cross-sections to next-to-leading order. Nucl. Phys., B467:399-442, 1996.

[19] Rikkert Frederix, Stefano Frixione, Fabio Maltoni, and Tim Stelzer. Automation of next-to-leading order computations in QCD: the FKS subtraction. JHEP, 10:003, 2009.

[20] R. Keith Ellis, D. A. Ross, and A. E. Terrano. The Perturbative Calculation of Jet Structure in e+ eAnnihilation. Nucl. Phys., B178:421, 1981.

[21] S. Catani and B.R. Webber. Infrared safe but infinite: Soft gluon divergences inside the physical region. JHEP, 9710:005, 1997.

[22] http://hepwww.rl.ac.uk/theory/seymour/nlo/event2_02.f. 